\title{
Contextual effects in difference judgments
}

\author{
HENDRIK N. J. SCHIFFERSTEIN \\ Agricultural University, Wageningen, The Netherlands
}

\begin{abstract}
Manipulating stimulus spacing, stimulus frequency, or stimulus range usually affects intensity judgments. In six experiments, I investigated the locus of analogues of these contextual effects in a "difference" estimation task. When all stimuli elicited the same taste quality, stimulus distribution affected the scale values only when water was included in the stimulus set (Experiments $1-3$ ). When the subjective ranges of two taste qualities were manipulated, different scale values were obtained for the separate qualities in the two conditions (Experiment 4). Manipulation of the expected response distribution did not affect the scale values or the responses (Experiments 5-6). It is concluded that shifts in stimulus distributions or stimulus ranges result in shifts in subjective scale values. The contextual effects can be interpreted as relative shifts of a number of gustatory continua, with water lying on a separate continuum. Proposed is a model for context-dependent judgments, consisting of four stages: stimulus classification, stimulus placement, continuum placement, and continuum projection.
\end{abstract}

A judgment of stimulus intensity represents the outcome of a number of physical, physiological, and psychological processes. Frijters (1993) distinguishes among three processing stages in single-stimulus judgment. First, a psychophysical function transforms the physical stimulus into a sensation. The sensation is regarded as the output from the sensory system. During perception, the intensity comes and goes gradually. The subject acts as a temporal averager, transforming a temporally variable sensory experience into a single impression-the coded sensation. During this process, attentive processes operate to enable the subject to focus upon the sensation attribute required by the experimental task. The stimulus is represented on an internal, subjective continuum and stored into working memory, where it may be accessed for judgmental processing. The third processing stage transfers the values of the coded sensations on the subjective continuum into responses on the response scale provided. This stage is called the judgment function, or the response output function.

Manipulating stimulus spacing, stimulus frequency, or stimulus range affects the mean intensity judgments of stimuli. When subjects are presented with many lowintensity stimuli, the ratings are higher than those when many high-intensity stimuli are presented. Examples of studies on sapid stimuli in which these contextual effects were reported are Riskey, Parducci, and Beauchamp (1979), Riskey (1982), McBride (1985), Schifferstein and Frijters (1992a), and Stillman (1993). It is still unclear which stage or stages of stimulus processing are responsible for contextual shifts in responses.

Address correspondence to H. N. J. Schifferstein, Department of Marketing and Marketing Research, Agricultural University, Bomenweg 2, 6703 HD Wageningen, The Netherlands (e-mail: rick.schifferstein@ alg.menm.wau.nl).

\section{Separating Sensory From Judgmental Processes}

One way to determine the locus or loci of contextual effects is by separating sensory from judgmental processes and determining which type of process is affected by the contextual shift. A separation of judgmental from sensory processes can be achieved by using a functional measurement framework in combination with a twostimulus procedure (Anderson, 1981; De Graaf, Frijters, \& van Trijp, 1987; Klitzner, 1975). In the two-stimulus procedure, subjects are instructed to estimate the size of a combination of two intensities elicited by two separate stimuli. The stimulus-processing sequence now contains an additional integration stage (Figure 1).

Stimulus pairs are constructed according to a full factorial judgment design: All stimuli from a series of first stimuli are combined with all stimuli from a series of second stimuli. After the experiment has been completed, mean responses are plotted as a function of the level of one stimulus, with a separate curve for each level of the other stimulus. A postexperimental statistical test determines whether the curves in the factorial plot are parallel. According to Anderson's (1981) parallelism theorem, a set of parallel curves in such a factorial plot implies that:

1. The subjects have used a linear additive integration rule. Examples of linear additive rules are addition $\left(r_{i j}=\right.$ $\left.s_{i}+s_{j}\right)$, subtraction $\left(r_{i j}=s_{i}-s_{j}\right)$, and averaging $\left(r_{i j}=\right.$ $s_{i}+s_{j} / 2$ ).

2 . The response output function is linear.

The postexperimental test, therefore, provides information on the cognitive integration rule and the response selection process, independently of the sensory processes that have occurred. After the linearity of the response output function and the type of cognitive integration rule have been checked and confirmed, the marginal means of the response matrix correspond to scale values for the (coded) sensations on an interval scale. 


\begin{tabular}{|c|c|c|c|}
\hline $\begin{array}{c}\text { Physical } \\
\text { zoncentration }\end{array}$ & $\begin{array}{l}\text { Perceived } \\
\text { sensation }\end{array}$ & $\begin{array}{c}\text { Coded } \\
\text { sensation }\end{array}$ & $\begin{array}{l}\text { Integrated } \\
\text { impression }\end{array}$ \\
\hline
\end{tabular}

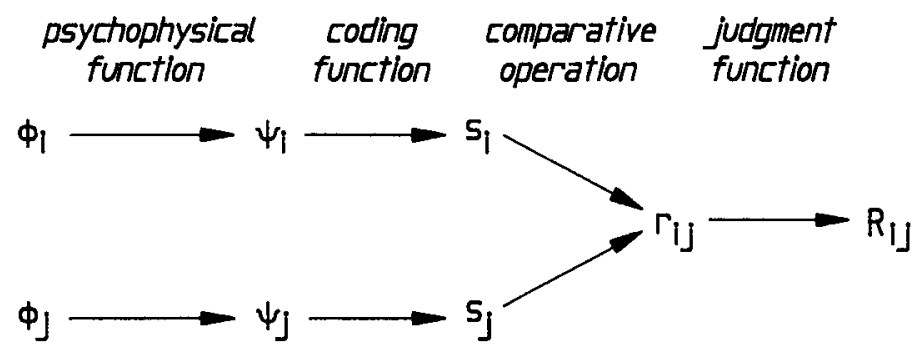

Figure 1. Conceptual outline of the two-stimulus procedure, following Frijters's (1993) three-stage model of psychophysical judgment. The subject compares the intensity of a sensation elicited by the first stimulus with the intensity elicited by the second stimulus.

De Graaf et al. (1987) have argued that the interval scale value of water is a conventional point of reference on a taste intensity scale that can be set equal to zero. If water does not elicit the target taste intensity, its scale value can be treated as an absolute zero point on a taste intensity scale. Consequently, De Graaf and Frijters (1988) suggested that the difference between the scale value of an experimental stimulus and the scale value for water gives a ratio scale value for the experimental stimulus.

Past research on context effects in two-stimulus tasks has mainly focused on the question of which process of stimulus judgment (Figure 1) is affected by the type of stimulus distribution (small-large range, positivelynegatively skewed). Changes in stimulus set composition could, theoretically, lead to shifts in responses by affecting either the psychological scale values of the stimuli (coded sensations), the cognitive integration process, or the form of the response output function.

The psychological scale values may be affected if changes occur in the perceptual process (e.g., due to sensory adaptation) or in the interpretation and encoding of the output from the sensory system. Changes in scale values will produce a factorial plot with a set of parallel curves differing in shape and vertical spacing between contexts (e.g., Mellers \& Birnbaum, 1982, their Figure 6). The type of cognitive integration rule (addition, averaging, or multiplication) is probably not affected by stimulus set composition. The integration function is usually considered to depend mainly on task instruction. If the stimulus context induces a nonlinear judgment function, the factorial plot gives a set of converging (negatively accelerating judgment function) or diverging (positively accelerating function) curves. If the judgment function remains linear, the curves remain parallel and the shapes of the curves and the relative distances between curves remain the same.

Birnbaum, Parducci, and Gifford (1971, Experiment 5) instructed subjects to judge the average line length of two lines on one card. When they combined their experimental stimuli with either a set of small line lengths (low filler) or a set of large line lengths (high filler), the factorial plot of the low-filler condition showed much more convergence than the high-filler plot (their Figure 8). According to these authors, the differences between conditions are due to changes in judgment functions and not to changes in scale values.

Mellers and Birnbaum (1982, Experiment 3) studied "total intensity" and "difference" judgments for the subjective size of a circle combined with the subjective darkness of a dot pattern. Four stimulus contexts yielded four different, parallel factorial plots for both task instructions. The differences between contexts are supposed to result from shifts in scale values. In Mellers and Birnbaum's Experiment 2, the subjects judged differences between the subjective darknesses of two dot patterns. Here, the difference judgments for the negatively and positively skewed stimulus distributions were similar, and the scale values were unaffected. These authors concluded that stimulus range and spacing influence the scale values underlying cross-modal judgments, whereas the type of stimulus distribution may affect only the judgment function in within-modal judgments.

\section{Manipulating Sensory and Judgmental Processes Independently}

Apart from the possibility of separating sensory and judgmental processes, the two-stimulus task allows independent manipulation of stimulus distribution and (expected) response distribution. In a single-stimulus intensity estimation task, a low-intensity stimulus is expected to yield a low response, and a high-intensity stimulus will yield a high response. In a two-stimulus "difference" estimation task, however, a small difference can be produced by either two low-intensity stimuli or two high-intensity stimuli. Departing from two identical stimulus sets, subjects can be presented with either many large differences or many small differences. By uncoupling stimulus intensity and response, one can investigate whether response selection tendencies (e.g., distributing the responses over the response continuum) are (partly) responsible for context effects. 


\section{The Present Study}

In the present study, contextual effects in a difference estimation task were investigated for the sense of taste. Experiments 1-3 employed positively or negatively skewed stimulus sets, composed of aqueous solutions of one substance only. In Experiment 4, unmixed solutions of two chemicals were used with different stimulus ranges. In Experiments 5 and 6, the expected response distribution was manipulated independently of the stimulus distribution.

\section{EXPERIMENT 1}

\section{Method}

Subjects. Twenty-four unpaid volunteers, 14 women and 10 men, ranging in age from 19 to 28 years, served as subjects. All the subjects were students of the Agricultural University, Wageningen. None of the subjects had had previous experience with psychophysical scaling experiments, and they all were naive with respect to the substance used and the purpose of the study.

Stimuli. The experimental stimuli were six solutions of sucrose (Merck 7651) in demineralized water: 0.0 (water), $0.1,0.2,0.4$, 0.8 , and 1.6 M. The reference pair consisted of 0.0 and $1.8 \mathrm{M}$. Solutions were prepared at least $24 \mathrm{~h}$ before tasting and were stored at $4^{\circ} \mathrm{C}$ for no longer than 4 days.

Design. Every condition contained one $6 \times 3$ full factorial judgment design. Each experimental stimulus was compared with three comparison stimuli: $0.0,0.4$, and $1.6 \mathrm{M}$ sucrose. In addition to the $6 \times 3=18$ pairs from the factorial judgment design, every subject was presented with a filler set of 12 pairs, containing either many lowconcentration stimuli (positive skew) or many high-concentration stimuli (negative skew). The positively skewed filler set consisted of 6 pairs $(0.1,0.1), 3$ pairs $(0.2,0.1), 1$ pair $(0.4,0.1), 1$ pair $(0.2$, $0.2)$, and 1 pair $(0.4,0.2)$. The negatively skewed filler set consisted of 6 pairs $(1.6,1.6), 3$ pairs $(1.6,0.8), 1$ pair $(1.6,0.4), 1$ pair $(0.8,0.8)$, and 1 pair $(0.8,0.4)$.

The pairs from the $6 \times 3$ factorial design (experimental stimuli presented first) were combined with one of the two filler sets described above and were presented to half the subjects. For the other subjects, the order of the two stimuli within each pair was reversed. Half the subjects received a positively skewed filler set, and the other half received a negatively skewed filler set. Consequently, 6 subjects served in each combination of stimulus position and skew.

Procedure. The subjects were instructed to judge the magnitude of the difference in perceived sweetness intensities between the first and the second stimulus of each pair. The instructions emphasized that only the sweetness intensity was to be judged, and that the hedonic value and side tastes were to be disregarded.

The judgments were expressed by a slash mark on a $250-\mathrm{mm}$ visual analogue scale. The middle of the scale was defined as "the first and second stimulus are equally sweet" (see De Graaf et al., 1987, their Figure 3). If the first stimulus was perceived as tasting sweeter than the second stimulus, the subject placed a mark on the left side of the scale, depending on the magnitude of the difference. The subject marked the right side of the scale when the second stimulus was perceived as being sweeter. The left end and the right end of the scale were labeled "maximum difference." In the instructions, maximum difference was defined as the difference in sweetness intensity between the stimuli of the reference pair - that is, water as the first stimulus and $1.8 \mathrm{M}$ of sucrose as the second stimulus. The difference between the stimuli of the reference pair was expected to be larger than the difference in any other pair. A response was measured as the distance in millimeters from the left pole of the scale. A response value of 125 meant no difference, a value above 125 meant that the second stimulus was perceived as sweeter than the first one, and a value below 125 indicated that the first stimulus was perceived as the sweetest of the pair.

The subjects were requested to rinse their mouths thoroughly with demineralized water both within and between pairs. The stimuli were presented at room temperature $\left(\sim 20^{\circ} \mathrm{C}\right)$ in polystyrene medicine cups. Each cup contained about $10 \mathrm{ml}$ of solution. The pairs were presented in a random sequence and in a different order for each subject. The reference pair was presented at the beginning of each session and after the 15 th pair. The time interval between and within pairs was $50 \mathrm{sec}$.

Statistical analysis. The "difference" responses for the full factorial designs were subjected to repeated measures analysis of variance (ANOVA). Three types of tests were performed.

1. The first test checked whether the order of presentation of the stimuli within pairs affected the results. If there is no significant main effect of stimulus order and no significant interaction, the data can be aggregated over stimulus orders.

2. After aggregation, the measure of the degree of nonparallelism - that is, the row stimulus $\times$ column stimulus interactionwas tested for significance. If this interaction term is not significant, it can be concluded that the subjects have used a subtractive comparative operation and a linear judgment function. Consequently, the marginal means of the row and column stimuli are valid estimates of the perceived taste intensity on an interval scale (Anderson, 1981). Assuming water is a rational zero point on the sweetness intensity scale, scale values were derived by calculating the difference between the marginal mean for each stimulus and the marginal mean for water (De Graaf et al., 1987).

3. Context effects were tested for significance by investigating the main effect of context and the context $X$ stimulus interactions.

Apart from statistical tests on the difference responses, the context effects were also investigated by repeated measures ANOVA of the individual scale values. These scale values were calculated from each individual's difference response matrix, as described above. Throughout this paper, $p \leq .05$ was used as the level of significance.

\section{Results}

Psychometric properties of response scale. In the ANOVA of the difference responses for the $6 \times 3$ full factorial design, neither the main effect of stimulus order nor any of the interactions yielded a significant $F$ value $(p>.10)$. Therefore, the data could be aggregated over the two stimulus orders. To test whether the subjects used a linear judgment function and a subtractive comparative operation, the measure of the degree of nonparallelismthat is, the experimental stimulus $\times$ comparison stimulus interaction-was tested. For the positive skew $[F(10,110)=1.55, p=.13]$ and the negative skew $[F(10,110)=1.33, p=.22]$, these tests were not significant. Consequently, scale values could be calculated.

Contextual effects. The two panels in Figure 2 are not identical; an ANOVA of the difference responses demonstrated that the type of stimulus distribution affected the responses by a significant skew $\times$ comparison stimulus interaction $[F(2,44)=7.79, p=.001]$. The main effect of skew $[F(1,22)=0.75, p=.40]$, the skew $\times$ experimental stimulus interaction $[F(5,110)=1.03, p=.40]$, and the skew $\times$ experimental $\times$ comparison stimulus interaction $[F(10,220)=1.16, p=.32]$ were not significant.

The calculated scale values show a substantial context effect for the comparison stimuli (Figure 3 ). The experimental stimuli show a similar trend: The ratio scale values in the positive skew exceed the scale values in the 
A. Positive skew

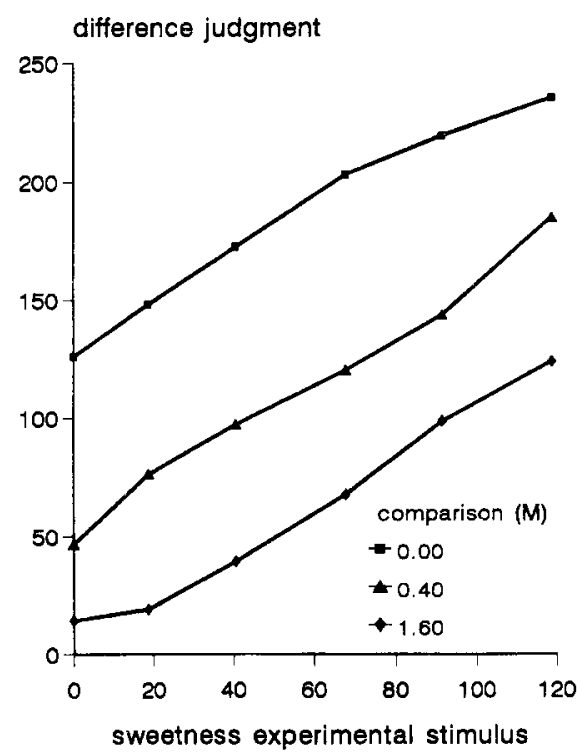

B. Negative skew

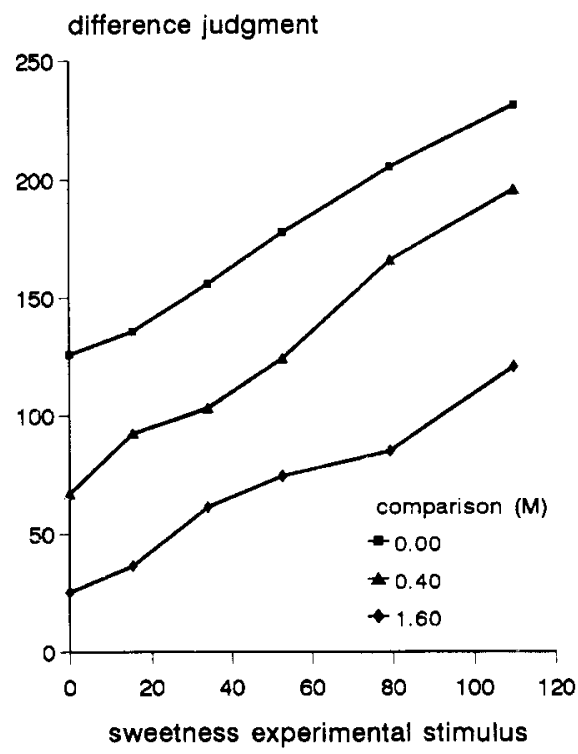

Figure 2. Mean reported judgment of difference in perceived sweetness intensities between the experimental stimulus and the comparison stimulus of each pair in Experiment 1. Panels $A$ and B show the mean responses for the positively skewed and negatively skewed stimulus distributions, respectively. The difference judgments are plotted as a function of the scale values for the experimental stimuli, with a separate curve for each comparison stimulus. Data were aggregated over stimulus sequences. They are plotted as if the comparison stimuli were always presented first in each pair.

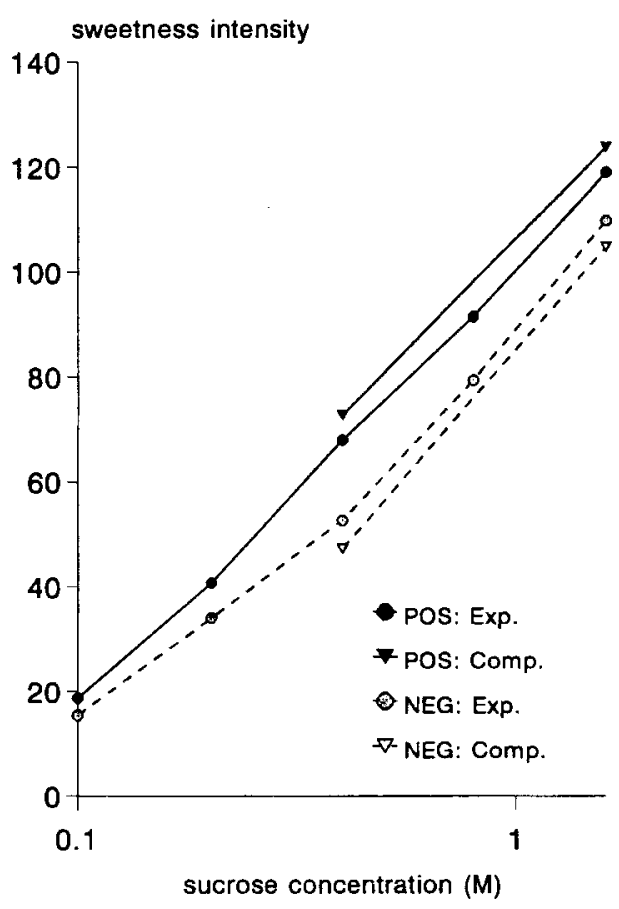

Figure 3. Scale values for the perceived sweetness intensities in Experiment 1. Separate curves are drawn for experimental (Exp.) and comparison (Comp.) stimuli presented in positively and negatively skewed stimulus distributions. negative skew. An ANOVA of the individual scale values showed a significant skew effect for the comparison stimuli $[F(1,22)=25.18, p<.001]$, but not for the experimental stimuli $[F(1,22)=2.86, p=.11]$. The curves obtained are essentially parallel. The skew $\times$ stimulus interaction was not significant in either ANOVA $(p>.40)$.

\section{EXPERIMENT 2}

Experiment 2 was designed to replicate the results of Experiment 1 for another taste quality. The design differed from Experiment 1 in that water was not included in the experimental stimulus set. Other differences are given below.

\section{Method}

Subjects. The subjects were 24 unpaid, naive volunteers, 16 women and 8 men, ranging in age from 19 to 26 years.

Stimuli. The experimental stimuli were four solutions of $\mathrm{NaCl}$ (Merck 6404) in demineralized water: $0.15,0.28,0.53$, and $1.0 \mathrm{M}$. The reference pair consisted of 0.0 (water) and $1.25 \mathrm{M}$ of $\mathrm{NaCl}$.

Design. Every condition contained one $4 \times 4$ full factorial judgment design in which each experimental stimulus was compared with all other experimental stimuli. In addition to the $4 \times 4=16$ pairs from the factorial judgment design, every subject was presented with a positively or negatively skewed filler set of 20 pairs The positively skewed filler set consisted of 10 pairs $(0.15,0.15)$, 4 pairs $(0.15,0.28), 4$ pairs $(0.28,0.15)$, and 2 pairs $(0.28,0.28)$. The negatively skewed filler set consisted of 10 pairs $(1.0,1.0), 4$ pairs $(0.53,1.0), 4$ pairs $(1.0,0.53)$, and 2 pairs $(0.53,0.53)$. Half 
the subjects received a positively skewed filler set, and the other half received a negatively skewed filler set.

Procedure. The subjects were instructed to judge the magnitude of the difference in perceived saltiness intensities between the first and the second stimulus of each pair. The reference pair was presented at the beginning of each session, after the 12th pair, and after the 24 th pair. The time interval between and within pairs was 40 sec.

\section{Results}

Psychometric properties of response scale. The left stimulus $X$ right stimulus interactions were tested for statistical significance in repeated measures ANOVAs of the $4 \times 4$ design. For the positive skew $[F(9,99)=0.97$, $p=.47]$ and the negative skew $[F(9,99)=0.96, p=$ $.48]$, these tests were not significant, implying parallelism in both panels of Figure 4.

Contextual effects. The type of stimulus distribution did not affect the difference responses, as demonstrated by insignificant $F$ values for the main effect of skew $[F(1,22)=3.55, p=.073]$ and for the skew $\times$ left stimulus $[F(3,66)=1.84, p=.15]$, skew $\times$ right stimulus $[F(3,66)=0.84, p=.48]$, and skew $\times$ left $\times$ right $[F(9,198)=0.80, p=.61]$ interactions.

Since water was not included in the stimulus set, the scale values were calculated as differences between the marginal mean for each stimulus and the marginal mean for $0.15 \mathrm{M}$ of $\mathrm{NaCl}$. These scale values show no sign of context effects (Figure 5). An ANOVA of the individual scale values revealed no significant effects of skew $[F(1,22)=0.00, p=.99]$ nor any skew $\times$ stimulus interaction $[F(2,44)=1.42, p=.25]$.

\section{A. Positive skew}

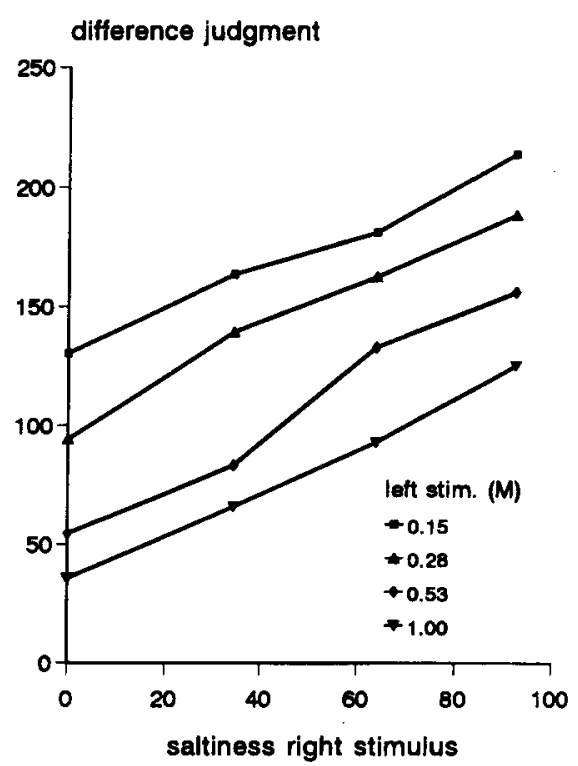

\section{Discussion}

The findings of Experiment 2 seem contradictory to those obtained in Experiment 1. However, the discrepancy disappears if the contextual effect in Figure 3 is explained as a shift in the interval scale value for water. The curves are essentially parallel, and if all scale values are calculated relative to $1.6 \mathrm{M}$ of sucrose, only one stimulus exhibits a context effect-water.

\section{EXPERIMENT 3}

Experiment 3 was a replication of Experiment 2, but it included water stimuli. In addition, the subjects evaluated a differently skewed distribution on a second occasion, 7 days later. Because the design was similar to that of Experiment 2, only specific differences are mentioned.

\section{Method}

Subjects. The subjects were 24 paid volunteers, 15 women and 9 men, ranging in age from 19 to 28 years. Eighteen subjects had previously participated in Experiment 4, Experiment 6 , or both (see below).

Stimuli. The experimental stimuli were five solutions of $\mathrm{NaCl}$ (Merck 6404) in demineralized water: 0.00 (water), 0.15, 0.28 , 0.53 , and $1.0 \mathrm{M}$. The reference pair consisted of 0.0 and $1.25 \mathrm{M}$ of $\mathrm{NaCl}$.

Design. Every condition contained one $5 \times 4$ full factorial judgment design, in which each experimental stimulus was compared with a set of comparison stimuli $(0,0.15,0.53,1.0 \mathrm{M})$. In addition to the $5 \times 4=20$ pairs from the factorial judgment design, every subject was presented with a positively or negatively skewed filler set of 16 pairs. The positively skewed filler set consisted of 7 pairs $(0.0,0.0), 4$ pairs $(0.0,0.15), 4$ pairs $(0.15,0.0)$, and 1 pair $(0.15$,

\section{B. Negative skew}

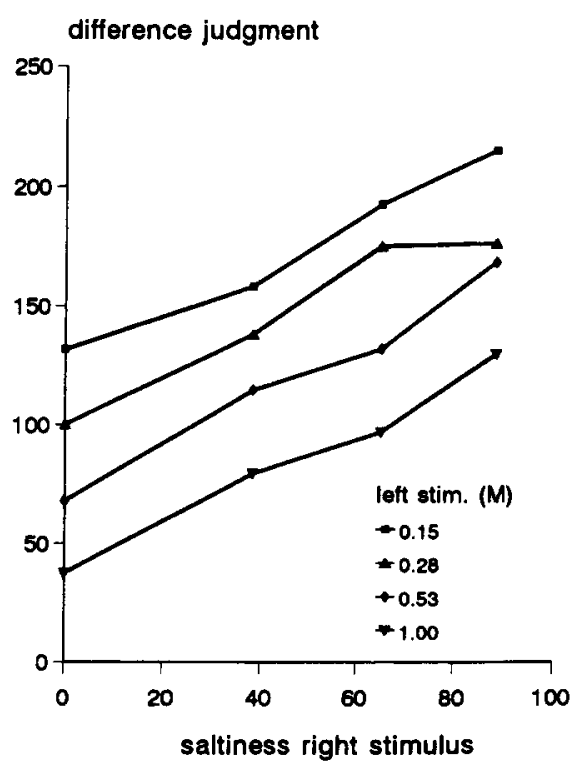

Figure 4. Mean reported judgment of difference in perceived saltiness intensities between the left and right stimulus of each pair in Experiment 2. Panels $A$ and $B$ show the mean responses for the positively skewed and negatively skewed stimulus distributions, respectively. The difference judgments are plotted as a function of the scale values for the right stimuli, with a separate curve for each left stimulus. 


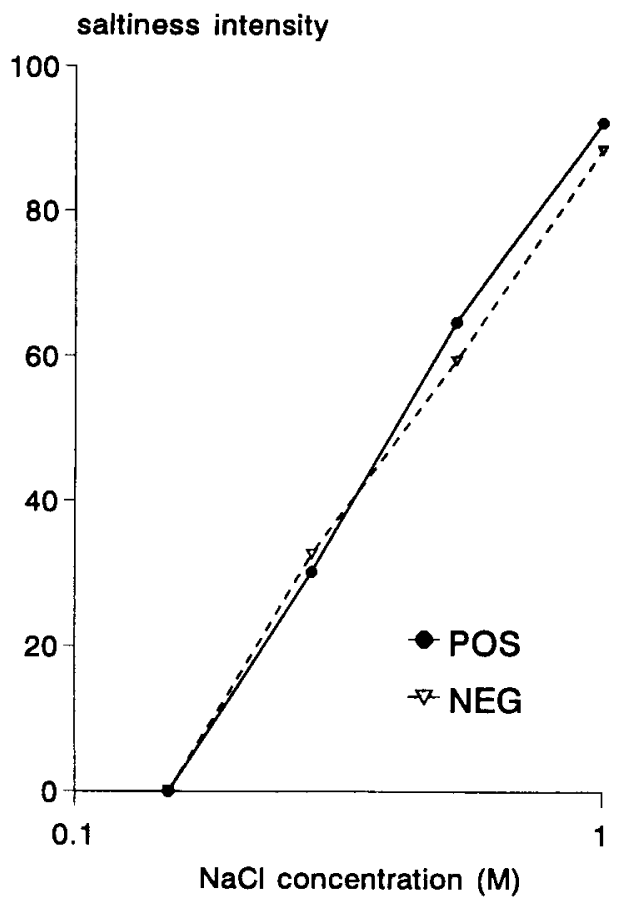

Figure 5. Scale values for the perceived saltiness intensities in Experiment 2. Separate curves are drawn for the positively and the negatively skewed stimulus distributions.

$0.15)$. The negatively skewed filler set consisted of 7 pairs (1.0, $1.0), 4$ pairs $(0.53,1.0), 4$ pairs $(1.0,0.53)$, and 1 pair $(0.53,0.53)$. Half the subjects received a positively skewed filler set, and the other half received a negatively skewed filler set. Within each condition, 6 subjects judged the $5 \times 4$ design (comparison on the right), and 6 subjects judged the $4 \times 5$ design (comparison on the left). One week later, the subjects who had evaluated the positively skewed stimulus distribution in Week 1 tasted the negatively skewed distribution, and vice versa.

\section{Results}

Psychometric properties of response scale. Repeated measures ANOVA of the $5 \times 4$ designs yielded no significant effect for the stimulus order within pairs nor any of its interactions for the data of the first $(p>.086)$ and the second week $(p>.10)$. Consequently, the data were aggregated over stimulus orders. The experimental $x$ comparison stimulus interaction was not significant for any of the conditions in the first or second week $(p>$ .15 ), implying parallel curves in Figure 6.

Contextual effects. In the first week, the stimulus distribution significantly affected the difference responses, as demonstrated by significant $F$ values for the skew $\times$ comparison stimulus $[F(3,66)=10.50, p<.001]$ and the skew $\times$ experimental stimulus $[F(4,88)=6.47, p<$ $.001]$ interactions. The main effect of skew $[F(1,22)=$ $1.13, p=.30]$ and the skew $\times$ experimental $\times$ comparison interaction $[F(12,264)=0.41, p=.96]$ were not significant. In the second week, the skew $\times$ comparison stimulus $[F(3,66)=2.50, p=.067]$ and the skew $\times$ experimental stimulus $[F(4,88)=2.53, p=.046]$ interactions were of borderline significance. The main effect of skew $[F(1,22)=1.73, p=.20]$ and the skew $\times$ comparison $\times$ experimental interaction $[F(12,264)=0.69$, $p=.76$ ] remained insignificant.

Scale values were calculated as differences between the marginal mean for each stimulus and the marginal mean for water. Figure 7 shows a substantial context effect for naive subjects: The mean difference in scale values between the two conditions equals 24 units. Withingroup comparisons of the scale values of Week 1 to those of Week 2 show an attenuation of the context effect: The mean difference is 14 units when the positive skew was sampled first, and 5 units when the negative skew was sampled first.

To find out whether the context effects reported can be ascribed to changes in the scale value for water, all the ANOVAs were calculated again for the responses in which no water stimuli were involved. These ANOVAs showed no significant main effect of skew and no significant interactions in the first week $(p>.20)$. In the second week, the skew $\times$ experimental stimulus interaction was significant $[F(3,66)=2.78, p=.048]$, whereas the other effects were not $(p>.14)$. In Figure 7, this significant effect appears as the differences in shapes of the two curves.

An ANOVA of the individual scale values showed significant effects for skew $[F(1,44)=7.4, p<.01]$ and a skew $\times$ week interaction $[F(1,44)=17.52, p<.001]$. The skew $\times$ stimulus interaction was also significant $[F(3,132)=2.84, p=.040]$, pointing at deviations from parallelism in Figure 7. This nonparallelism is due to shifts in scale values in the second week: In contrast to the data for the second week $[F(3,66)=3.61, p=$ $.02]$, the naive subjects showed no skew $\times$ stimulus interaction $[F(3,66)=0.98, p=.41]$.

\section{Discussion}

Experiments 1-3 show that contextual shifts in scale values occur only when water is included in the qualitatively homogeneous stimulus set. This finding allows for two interpretations that are mathematically equivalent.

1. If the scale value for water is used as a zero point (De Graaf et al., 1987), it follows that the perceived stimulus intensities differ between the two stimulus distributions.

2. Only the scale value for water is affected by manipulations of stimulus context. The scale values for the sucrose or $\mathrm{NaCl}$ solutions are roughly similar in the two contexts.

Experiments 1-3 point out that the scale value for water or the zero point on the internal, subjective taste intensity continuum plays an important role in contextual effects. When many low-intensity stimuli are presented, the subjective difference in taste intensity between the "no-taste" (water) stimuli and the "taste" (sucrose or $\mathrm{NaCl}$ ) stimuli increases. The effects of the skewed stimulus distributions are absent when only "taste" stimuli are presented (Experiment 2).

Mellers and Birnbaum (1982) also found no sign of context effects when subjects rated the "differences" or 
A. POS week 1

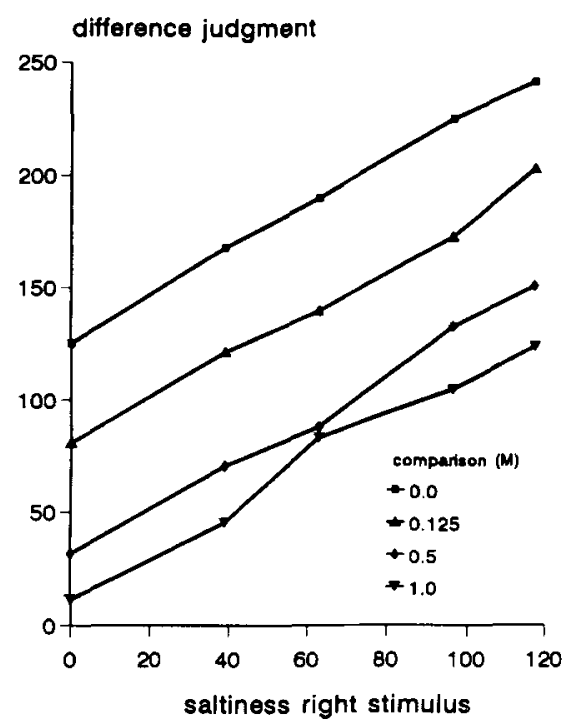

C. POS week 2

difference judgment

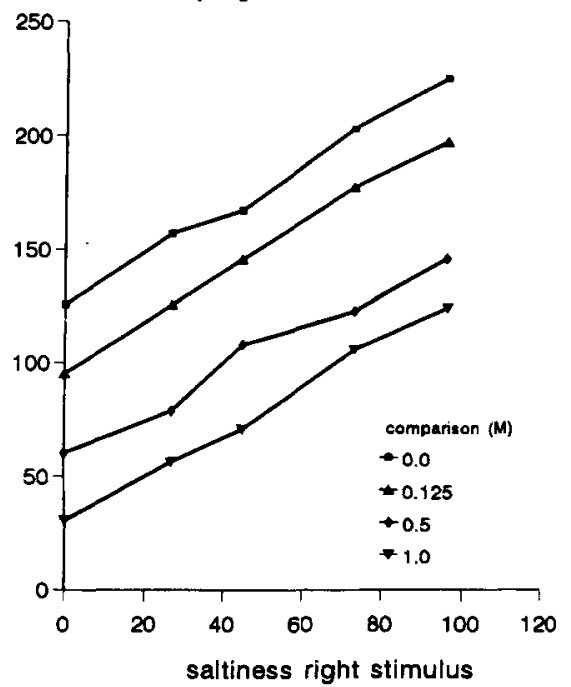

B. NEG week 1

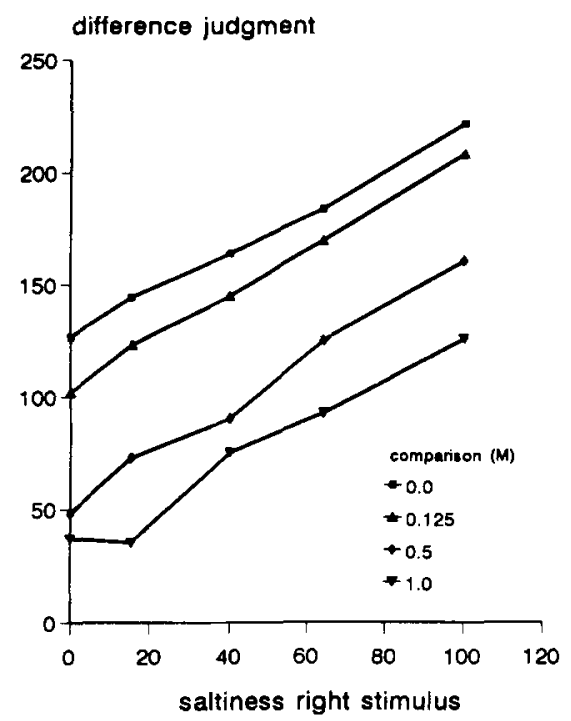

D. NEG week 2

difference judgment

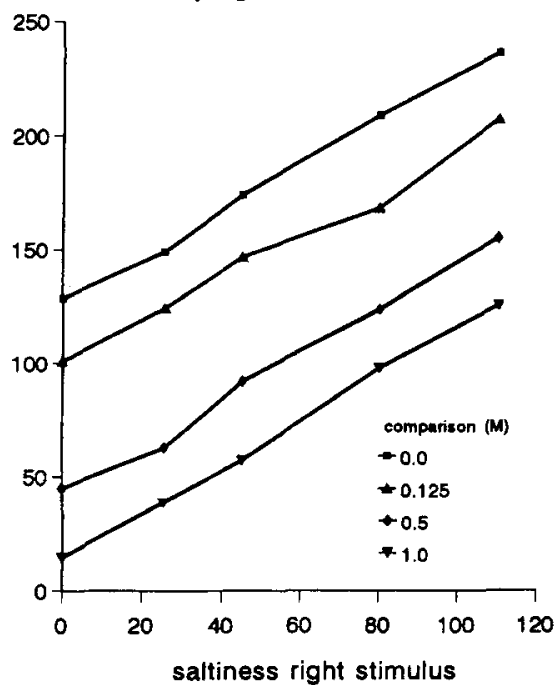

Figure 6. Mean reported judgment of difference in perceived saltiness intensities between the left and right stimulus of each pair in Experiment 3. Panels $A$ and $B$ show the mean responses for the positively skewed and negatively skewed stimulus distributions for naive subjects. Panels $C$ and $D$ show mean responses for subjects who received a differently skewed distribution the week before. The difference judgments are plotted as a function of the scale values for the experimental stimuli, with a separate curve for each comparison stimulus. Data were aggregated over stimulus sequences. They are plotted as if the comparison stimuli were always presented first in each pair.

"ratios" of darknesses of dot patterns of stimulus sets with no blanks. These authors found shifts in scale values only in a cross-modal comparison task. They argued that each half of a stimulus pair is first compared within the stimulus set of its own modality, before it is compared with the stimuli from the other modality. Marks and colleagues have referred to this phenomenon as "loose coupling" between stimulus continua (e.g., Marks
\& Warner, 1991; Rankin \& Marks, 1991). The present findings suggest that subjects do not regard the blank stimuli as part of the target taste continuum.

Figure 7 shows that scale values in the second week are a compromise between the values that naive subjects attribute to the stimuli in the two stimulus distributions. A transfer of stimulus context has been previously reported with shifts in stimulus range (Stillman, 1993; 


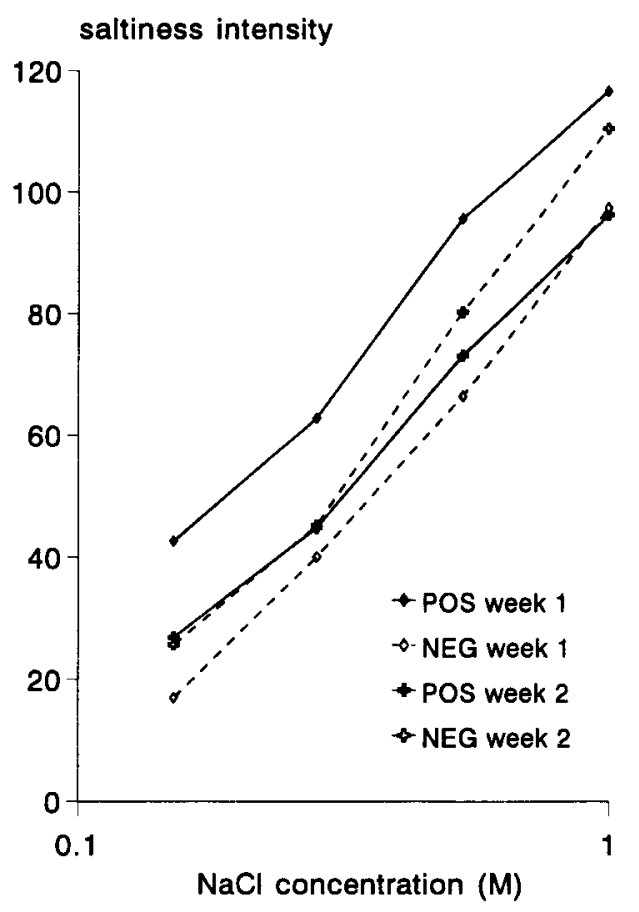

Figure 7. Scale values for the perceived saltiness intensities in Experiment 3. The line style indicates whether the stimulus distribution was positively (solid lines) or negatively (dotted lines) skewed. The type of symbol indicates whether the results were obtained in the first (diamonds) or second (crosses) week.

Ward, 1987; Ward \& Lockhead, 1970) and stimulus frequency (Schifferstein, 1994a) in single-stimulus tasks.

\section{EXPERIMENT 4}

Rankin and Marks (1991) have studied the coupling between the sweetness and the saltiness continuum using a dual-context paradigm. One stimulus set consisted of a set of high concentrations of sucrose and low concentrations of $\mathrm{NaCl}$. The other stimulus set contained high concentrations of $\mathrm{NaCl}$ and low concentrations of sucrose. Subjects judged the total taste intensity of one stimulus set by using free modulus magnitude estimation. These two conditions yielded different intensity estimates and different "equi-intense" sucrose- $\mathrm{NaCl}$ matches.

The differences in matches argue in favor of different scale values for $\mathrm{NaCl}$ and sucrose in the two conditions. However, since magnitude estimates are the joint result of sensory, cognitive, and judgmental processes, the stimulus matches could also result from different response output functions for sweet and salty stimuli. In Experiment 4, I investigated whether loose coupling of stimulus continua can be demonstrated by using the difference estimation task. The two-stimulus task separates the response output function from the scale values and, thereby, eliminates the possibility of different response output functions. Specific differences with the previous experiments are mentioned below.

\section{Method}

Subjects. Twenty-four paid volunteers, 14 women and 10 men, ranging in age from 19 to 28 years, served as subjects. Thirteen subjects had previously participated in Experiment 6 (see below).

Stimuli. The experimental stimuli were solutions of sucrose (Merck 7651) and $\mathrm{NaCl}$ (Merck 6404) in demineralized water. The sucrose and $\mathrm{NaCl}$ concentrations were 0.0 (water), 0.0313 , $0.0625,0.125,0.25,0.5$, and $1.0 \mathrm{M}$. The reference pair consisted of 0.0 and $1.25 \mathrm{M}$ of sucrose or $\mathrm{NaCl}$.

Design. The two conditions were constructed from two $5 \times 5$ full factorial judgment designs. In the $\mathrm{S}_{\mathrm{L}} \mathrm{N}_{\mathrm{H}}$ condition, the sucrose concentrations were low $(0.0,0.0313,0.0625,0.125,0.25 \mathrm{M})$, and the $\mathrm{NaCl}$ concentrations were high $(0.0,0.125,0.25,0.5,1.0 \mathrm{M})$. In the $\mathrm{S}_{\mathrm{H}} \mathrm{N}_{\mathrm{L}}$ condition, the sucrose concent rations were high $(0,0$, $0.125,0.25,0.5,1.0)$, and the $\mathrm{NaCl}$ concentrations were low $(0.0$, $0.0313,0.0625,0.125,0.25)$. Identical stimuli in both conditions were water, $0.125 \mathrm{M}$ of $\mathrm{NaCl}, 0.25 \mathrm{M}$ of $\mathrm{NaCl}, 0.125 \mathrm{M}$ of sucrose, and $0.25 \mathrm{M}$ of sucrose. Every subject received one $5 \times 5$ design twice. In one design, the sucrose stimuli were on the left and the $\mathrm{NaCl}$ stimuli were on the right. In the replication, the order of the stimuli within pairs was reversed. The subject tasted the 50 pairs in random order.

Procedure. The subjects were instructed to judge the magnitude of the difference in total taste intensities between the first and the second stimulus of each pair. The instructions emphasized that every perceived quality was to be included in the total intensity judgment and that qualitative differences had to be ignored.

In the $\mathrm{S}_{\mathrm{L}} \mathrm{N}_{\mathrm{H}}$ condition, water and $1.25 \mathrm{M}$ of $\mathrm{NaCl}$ were used as references denoting the maximum intensity difference. In the $\mathrm{S}_{\mathrm{H}} \mathrm{N}_{\mathrm{L}}$ condition, water and $1.25 \mathrm{M}$ of sucrose were used as the reference pair. The references were presented at the beginning of each session and again after the $12 \mathrm{th}, 25 \mathrm{th}$, and $37 \mathrm{th}$ pair. The time interval between and within pairs was $30 \mathrm{sec}$.

\section{Results}

Psychometric properties of response scale. First, the data of each condition were subjected to a $2 \times 5 \times 5 \times 12$ ANOVA (stimulus order $\times$ sucrose $\times \mathrm{NaCl} \times$ subjects) to check whether the order of presentation of the stimuli within pairs had affected the results. For both conditions, the main effect of stimulus order and the interactions with the most intense stimulus series were highly significant $(p<.01)$. The three-way interactions (stimulus order $\times$ sucrose $\times \mathrm{NaCl})$ were also significant $(p \leq .05)$. When sucrose is presented as first stimulus, the intensity of the sucrose stimulus seems smaller and the intensity of the $\mathrm{NaCl}$ stimulus seems larger than when sucrose is the second stimulus (Figure 8). On the average, the scale value for a right stimulus was 12.9 units larger than that for a left stimulus.

Despite these differences, the data were aggregated to investigate the effects of stimulus context on scale values and the form of the response output function. An ANOVA yielded no significant sucrose $\times \mathrm{NaCl}$ interactions $(p>.17)$, supporting parallelism. Scale values were calculated from the marginal means (Figure 9).

Contextual effects. An ANOVA of the common $3 \times$ 3 design $(0.00,0.125,0.25 \mathrm{M}$ of $\mathrm{NaCl} \times 0.00,0.125$, $0.25 \mathrm{M}$ of sucrose) demonstrated that context affected the difference responses. The main effect of range $[F(1,22)=25.81, p<.001]$ and the range $\times \mathrm{NaCl}$ $[F(2,44)]=11.26, p<.001]$ and the range $\times$ sucrose interactions $[F(2,44)=3.81, p=.030]$ were all signif- 
A. Sucrose: low, left

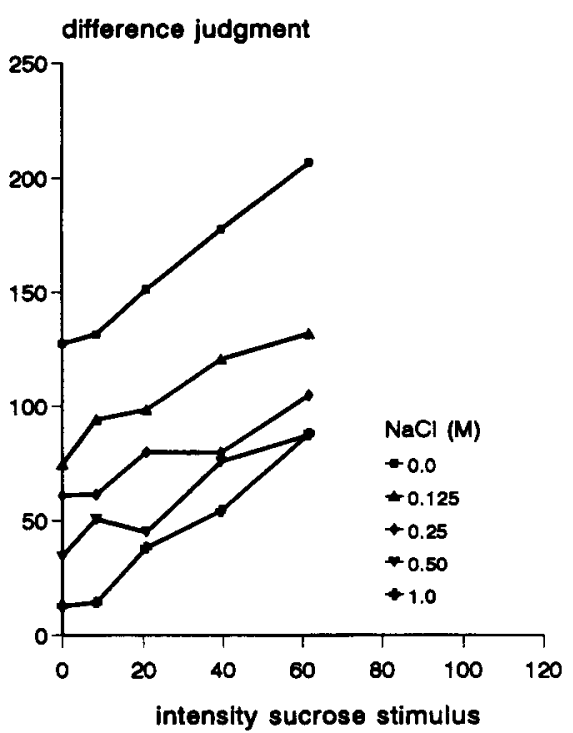

C. Sucrose: high, left

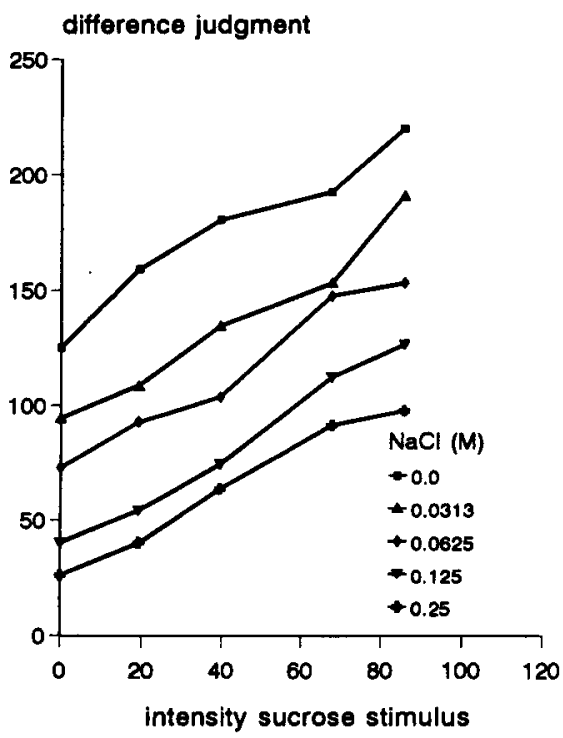

B. Sucrose: low, right

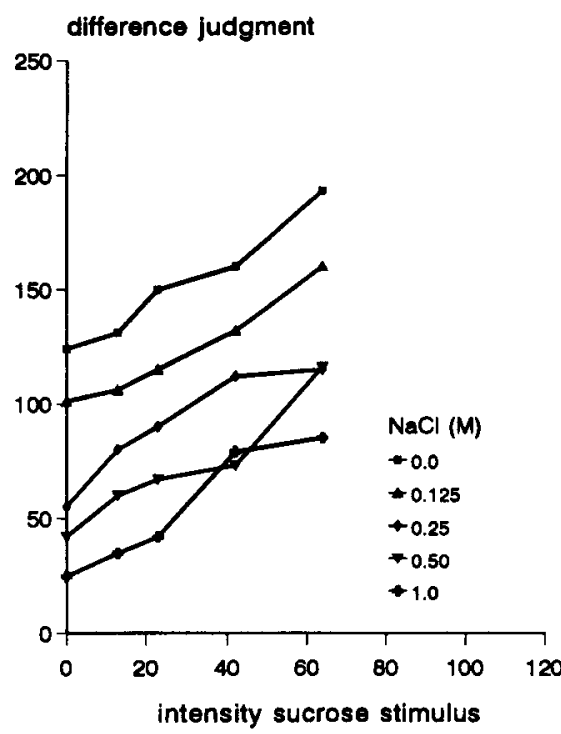

D. Sucrose: high, right

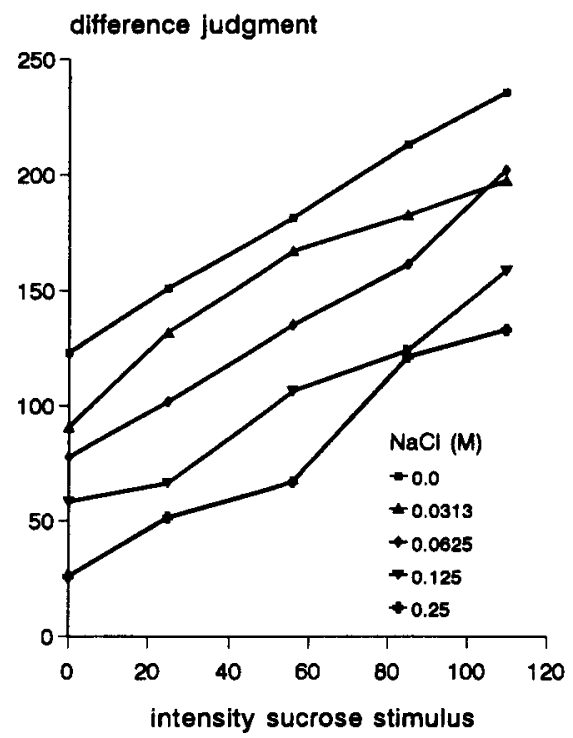

Figure 8. Mean reported judgment of difference in perceived total taste intensities between the left and right stimulus of each pair in Experiment 4. The first two panels show the mean responses in the $S_{L} N_{H}$ condition for sucrose presented as the first (panel $A$ ) or second (panel B) stimulus of each pair, respectively. The other panels show the mean responses in the $S_{H} N_{L}$ condition for sucrose presented as the first (panel $C$ ) or second (panel D) stimulus of each pair, respectively. The data in panels $A$ and $C$ were transformed by the formula $R^{\prime}=250-R$ to facilitate comparison with panels $B$ and $D$. The difference judgments are plotted as a function of the scale values for the sucrose stimuli, with a separate curve for each NaCl stimulus.

icant. The three-way interaction was not $[F(4,88)=$ $0.43, p=.79]$.

An ANOVA of the individual scale values yielded significant range effects $[F(1,22)=20.8, p<.001$ for $\mathrm{NaCl} ; F(1,22)=4.73, p=.04$ for sucrose]. The absence of range $X$ stimulus interactions $[F(1,22)=3.14, p=$ .09 for $\mathrm{NaCl} ; F(1,22)=0.77, p=.39$ for sucrose $]$ indi- cates parallel sucrose curves and parallel $\mathrm{NaCl}$ curves in Figure 9.

The scale values for sucrose and $\mathrm{NaCl}$ exhibit substantial shifts between conditions. "Equi-intense" matches differ between conditions. In Figure 9, $0.125 \mathrm{M}$ of $\mathrm{NaCl}$ and $0.125 \mathrm{M}$ of sucrose have similar scale values in the $\mathrm{S}_{\mathrm{L}} \mathrm{N}_{\mathrm{H}}$ condition. In the $\mathrm{S}_{\mathrm{H}} \mathrm{N}_{\mathrm{L}}$ condition, however, the 


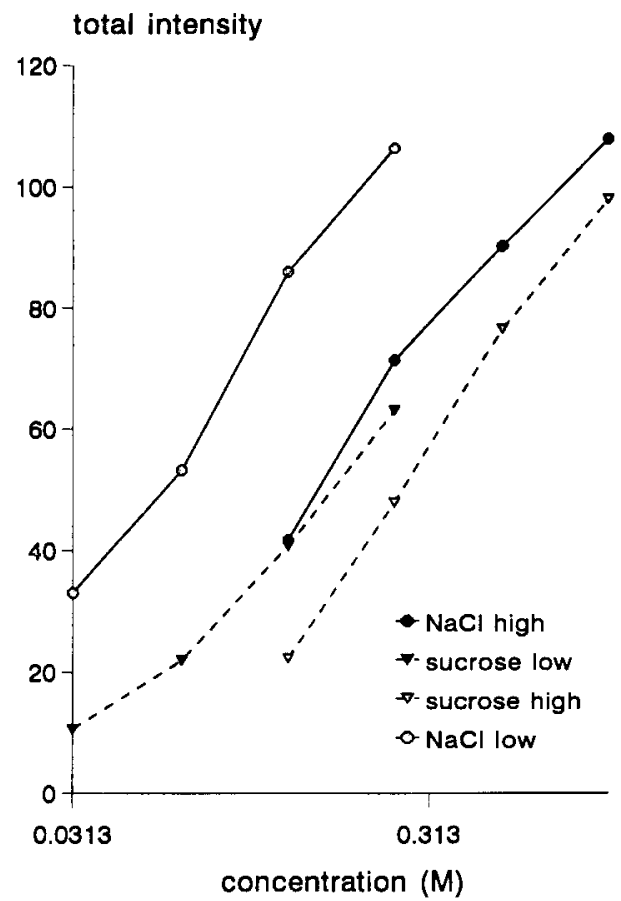

Figure 9. Scale values for the perceived total taste intensities in Experiment 4. The line style indicates whether the stimulus was $\mathrm{NaCl}$ (solid lines) or sucrose (dotted lines). The open symbols show the results for the $S_{H} N_{L}$ condition, and the closed symbols show those for the $S_{L} N_{H}$ condition.

stimulus match for $0.125 \mathrm{M}$ of $\mathrm{NaCl}$ lies between 0.5 and $1.0 \mathrm{M}$ of sucrose.

\section{Discussion}

Again, the contextual effects observed for the scale values calculated with water as the reference point can also be interpreted as resulting from shifts in the scale value for water. In this case, each continuum (salty, sweet) must have its own, context-dependent scale value for water. The present results show that the shifts in $\mathrm{NaCl}$-sucrose matches reported by Rankin and Marks (1991) are not due to different response output functions for $\mathrm{NaCl}$ and sucrose, but result from contextdependent internal representations. Schneider and Parker (1990) and Marks (1992) reached a similar conclusion employing auditory stimuli in nonnumerical judgment tasks.

\section{EXPERIMENT 5}

In Experiment 5, I investigated whether contextual effects can be explained as a response selection effect. In this experiment, the response distribution was manipulated independently of the stimulus distribution.

\section{Method}

Subjects. The subjects were 24 unpaid, naive volunteers, 12 women and 12 men, ranging in age from 20 to 26 years.
Stimuli. The experimental stimuli were solutions of fructose (Merck 5321) in demineralized water: 0.00 (water), $0.15,0.30$, and $0.60 \mathrm{M}$. The reference pair consisted of 0.00 and $0.75 \mathrm{M}$ of fructose.

Design. Every condition contained one $4 \times 4$ full factorial judgment design, in which each experimental stimulus was compared with all the other experimental stimuli. In addition to the 16 pairs from the factorial judgment design, every subject was presented with a filler set of 20 pairs. In this experiment, not the absolute intensities, but the differences in intensities within pairs were skewed: In the SD filler set, the differences between the two samples were small, and in the LD filler set, they were large.

The stimulus concentrations in this experiment were selected to yield approximately equal intensity differences between consecutive concentration levels. These intensity differences were used to obtain two skewed distributions of differences $(\Delta)$. The $4 \times 4$ full factorial design consisted of four pairs with identical stimuli $(\Delta=0)$, six pairs where $\Delta=1$, four pairs where $\Delta=2$, and two pairs where $\Delta=3$. The combination of the $4 \times 4$ design with either filler set yielded a positively or negatively skewed distribution of intensity differences (Tables 1 and 2). The stimulus sets were equal in both conditions: 0.00 and $0.60 \mathrm{M}$ were presented 25 times, $0.15 \mathrm{M}$ and $0.30 \mathrm{M}$ were presented 11 times.

Procedure. The procedure was similar to the one used in Experiment 1 . The reference pair was presented at the beginning of each session, after the 12 th pair, and after the 24 th pair. The time interval between samples was $40 \mathrm{sec}$.

\section{Results}

Psychometric properties of response scale. The left $x$ right stimulus interaction was tested in an ANOVA of the $4 \times 4$ design. For the SD condition $[F(9,99)=0.71, p=$ $.70]$ and the LD condition $[F(9,99)=1.87, p=.07]$, these tests were not significant. Consequently, panels $A$ and $\mathrm{B}$ in Figure 10 exhibit parallelism. Scale values are shown in Figure 11.

Contextual effects. The type of difference distribution did not affect the responses, as demonstrated by nonsignificant $F$ values for the main effect of skew $[F(1,22)=$ $1.89, p=.18]$ and skew $\times$ left stimulus $[F(3,66)=$

Table 1

Distribution of Stimulus Differences for Experiments 5 and 6

\begin{tabular}{lrrrr}
\hline & \multicolumn{4}{c}{ Size of Difference $(\Delta)$} \\
\cline { 2 - 5 } & 0 & 1 & 2 & 3 \\
\hline $4 \times 4$ design & 4 & 6 & 4 & 2 \\
SD filler & 12 & 4 & 2 & 2 \\
LD filler & 0 & 0 & 6 & 14 \\
\hline $4 \times 4+$ SD & 16 & 10 & 6 & 4 \\
$4 \times 4+$ LD & 4 & 6 & 10 & 16 \\
\hline
\end{tabular}

Note--SD, small differences; LD, large differences.

Table 2

Frequency of Presentation of Pairs for the Conditions Employing the SD Filler Set or the LD Filler Set

\begin{tabular}{|c|c|c|c|c|c|c|c|c|}
\hline \multirow[b]{3}{*}{ Left Stimulus } & \multicolumn{8}{|c|}{ Right Stimulus } \\
\hline & \multicolumn{4}{|c|}{ SD Filler Condition } & \multicolumn{4}{|c|}{ LD Filler Condition } \\
\hline & 0.00 & 0.15 & 0.30 & 0.60 & 0.00 & 0.15 & 0.30 & 0.60 \\
\hline 0.00 & 7 & 2 & 2 & 2 & 1 & 1 & 2 & 8 \\
\hline 0.15 & 2 & 1 & 1 & 2 & 1 & 1 & 1 & 3 \\
\hline 0.30 & 1 & 1 & 1 & 2 & 3 & 1 & 1 & 1 \\
\hline 0.60 & 2 & 1 & 2 & 7 & 8 & 2 & 1 & 1 \\
\hline
\end{tabular}

Note-SD, small differences; LD, large differences. 
A. SD Exp. 5

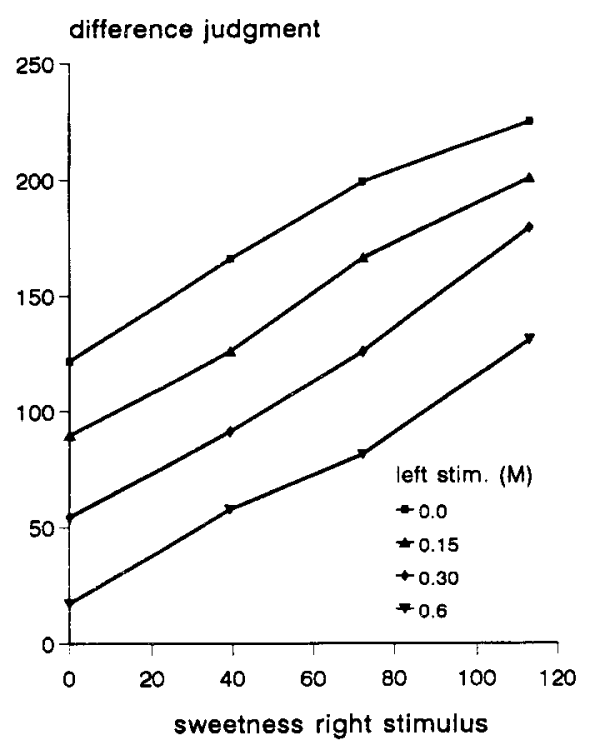

C. SD Exp. 6

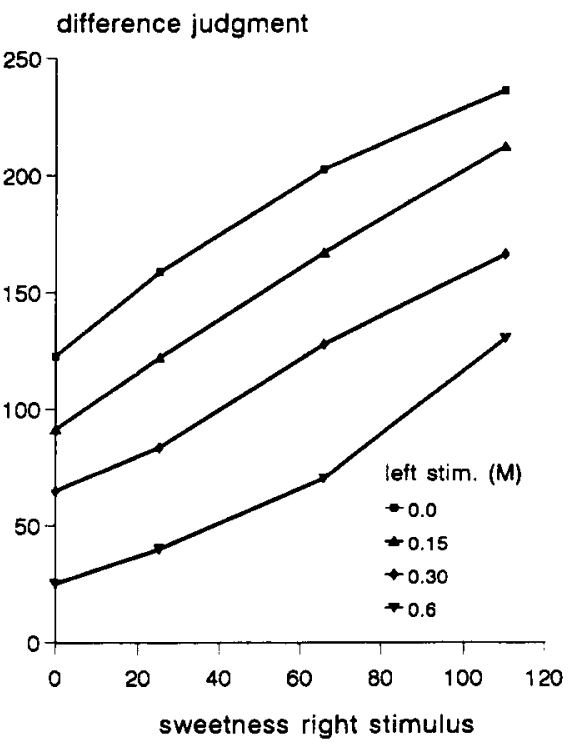

B. LD Exp. 5

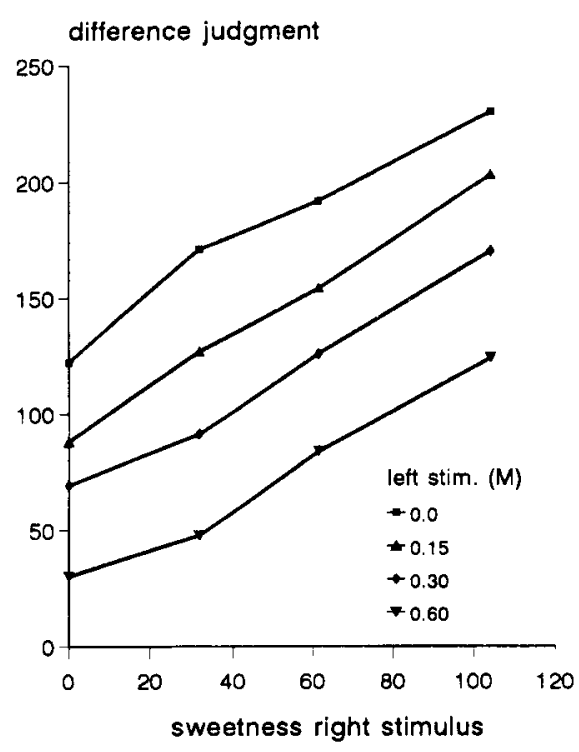

D. LD Exp. 6

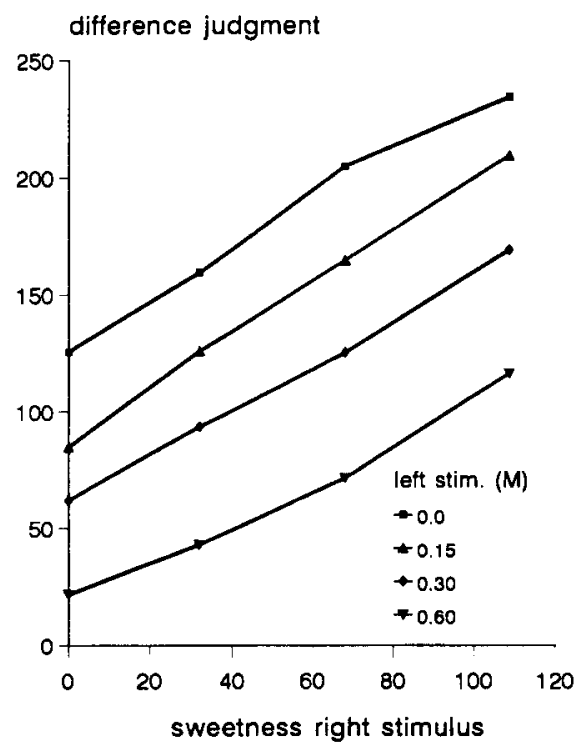

Figure 10. Mean reported judgment of difference in perceived sweetness intensities between the left and right stimulus of each pair in Experiments 5 (panels A-B) and 6 (panels C-D). In both experiments, subjects were presented with either many small intensity differences (SD: panels $A$ and $C$ ) or many large intensity differences (LD: panels B and D). The difference judgments are plotted as a function of the scale values for the right stimuli, with a separate curve for each left stimulus.

$0.08, p=.97]$, skew $\times$ right stimulus $[F(3,66)=0.77$, $p=.52]$, and skew $\times$ left $\times$ right $[F(9,198)=0.73$, $p=.68]$ interactions.

\section{EXPERIMENT 6}

Experiment 5 showed no effect of response distribution on the mean difference responses. It can be ar- gued that the scaling method employed in Experiment 5 was not optimal to demonstrate such an effect, because a large difference could result in a response on the outer left part (left $»$ right) or on the outer right part (right " left) of the scale. Therefore, the distribution of differences was not one-to-one related to a specific location on the response scale. Experiment 6 was a replication of Experiment 5 with a slightly dif- 


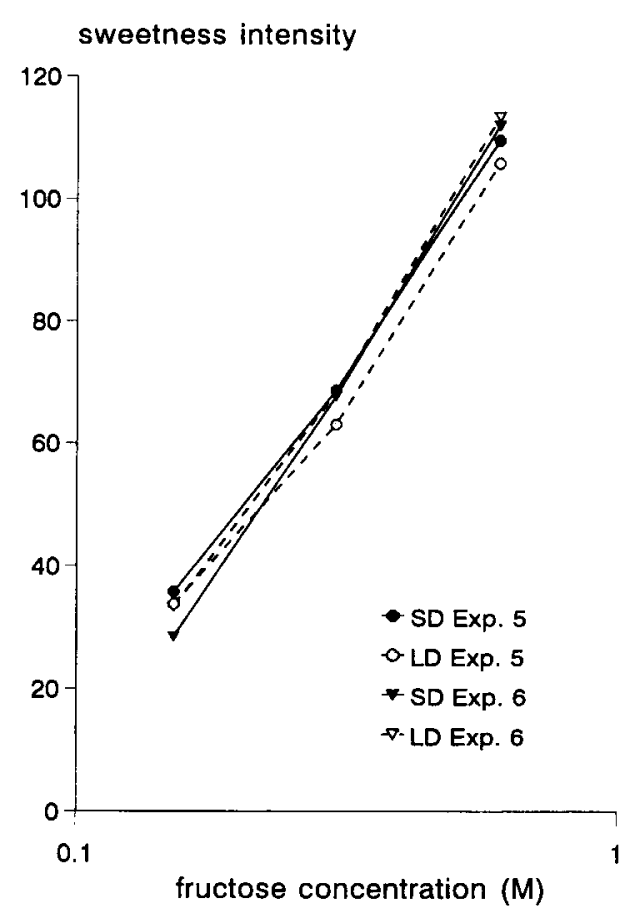

Figure 11. Scale values for the perceived sweetness intensities in Experiments 5 and 6 . The line style distinguishes between the two experiments. The closed symbols indicate the small-differences (SD) conditions, and the open symbols indicate the large-differences (LD) conditions.

ferent rating scale; specific differences are mentioned below.

\section{Method}

Subjects. The subjects were 24 paid, naive volunteers, 16 women and 8 men, ranging in age from 19 to 25 years.

Procedure. In contrast to the previous experiments, the subjects scaled the sizes of the perceived sweetness intensity differences on 125-mm line scales. The left end of the scale was labeled "the left and right stimulus are equally sweet." The right end of the scale was labeled "maximum difference." For each pair in which a difference was perceived, the subjects indicated whether the left or right stimulus was the sweetest, by circling an $L$ or an $R$ on the form

\section{Results}

Psychometric properties of response scale. The left stimulus $X$ right stimulus interaction was tested for statistical significance in a repeated measures ANOVA of the $4 \times 4$ design. For the SD condition $[F(9,99)=2.09$, $p=.038]$ and the LD condition $[F(9,99)=2.55, p=$ $.011]$, the tests were significant. Instead of a set of parallel curves, the factorial plots are somewhat barrel shaped (Figures 10C and 10D). To investigate whether these significant deviations affected the scale values, the relative magnitude of experimental effects $\left(\omega^{2}\right)$ was calculated for a three-way mixed-model design (Vaughan \& Corballis, 1969). Since the sizes of the experimental left $\times$ right effects were small $(0.63 \%$ and $0.37 \%$ for the SD and LD conditions, respectively), the effect of the deviations from parallelism on the scale values is negli- gible. Therefore, scale values were calculated in the usual way (Figure 11).

Contextual effects. As in the previous experiment, the type of difference distribution did not affect the responses. The main effect of skew $[F(1,22)=0.9, p=.37]$ and the skew $\times$ left stimulus $[F(3,66)=0.29, p=.83]$, skew $\times$ right stimulus $[F(3,66)=0.64, p=.59]$, and skew $\times$ left $\times$ right $[F(9,198)=0.74, p=.67]$ interactions were not significant.

A comparison of Experiments 5 and 6. An overall ANOVA on the results of Experiments 5 and 6 yielded insignificant $F$ values for the main effect of experiment and all its interactions $(p>.06)$. Apparently, both response scales yield approximately the same results. Also, the scale values in both experiments are similar (Figure 11).

\section{GENERAL DISCUSSION}

\section{Contextual Effects in One- and Two-Stimulus Procedures}

When using findings obtained in a difference estimation task to explain the outcomes of single-stimulus scaling experiments, one assumes that psychophysical functions and coding functions are similar under the two procedures. Critics may conjecture that the representation of the first stimulus in the difference task may deviate from the representation of the second stimulus, since the former is retained in memory for an additional interstimulus interval. However, the absence of stimulus order effects and the good correspondence between the intensity estimates from one- and two-stimulus procedures in "homogeneous" stimulus sets (De Graaf, Legger, \& Roozen, 1990) support the assumption. Consequently, the present findings are discussed below in relation to single-stimulus tasks.

The contextual shifts in scale values resemble the shifts in ratings obtained with a single-stimulus procedure. In contrast with the difference estimation task, however, shifts in ratings occur even when water is not an experimental stimulus (e.g., Riskey et al., 1979; Schifferstein \& Frijters, 1992a). The context effect may occur because subjects in a single-stimulus procedure need some internal references for "no taste" and "extremely strong taste" to make their judgments. Even though water may not be presented as an experimental stimulus, it is often present as rinsing-water and may thus help to establish a subjective, context-dependent frame of reference.

A difference between context effects in one- and twostimulus procedures lies in the shape of the obtained relationships between stimulus and (inferred) intensity. A single-stimulus procedure usually produces two curves for the negative skew and the positive skew that converge at either end: The contextual shift is smallest for extreme stimulus values. With the two-stimulus procedure, however, the curves are essentially parallel. Possibly, the overall shift in rating for the one-stimulus procedure results from a shift in internal representation, 
whereas the change in the form of the stimulus-response relationship results from a change in judgment function. This supposition concurs with one of my previous reports (Schifferstein, 1994b), where a significant correlation between the degree of response variability and the size of the context effect suggested that context effects for judgments of unmixed stimuli are (partially) due to response selection processes.

\section{Within-Set Contextual Effects}

Experiment 4 showed that scale values for second stimuli were, on the average, 12.9 units larger than those for first stimuli. Such a large deviation seems typical for the "heterogeneous" sweet-salty pairs. The mean deviation varied from -4.4 to 5.2 in the five "homogeneous" sweet-sweet and salty-salty experiments. The observed discrepancy may result from successive contrast: After tasting a sweet solution, the salty taste stands out against the sweet sensation and is, consequently, overestimated (e.g., Kroeze, 1983; Schifferstein \& Frijters, 1992b).

\section{The Locus of Contextual Effects}

For complex stimulus sets in which two or more stimulus domains can be distinguished, Parducci, Knobel, and Thomas (1976) showed that ratings for one stimulus domain can be made independently of the stimulus distribution in the other domain. Since the context effects appear for each stimulus domain independently of the responses on the common response continuum, they suggested that subjects count or represent stimulus frequencies rather than response frequencies: "Subjects are putting the same number of stimuli in each category rather than using their alternative response categories with equal frequency" (p. 365). Parducci et al.'s suggestion is supported by the results of Experiments 5 and 6 : Judgments are not affected by the number of times a response is used on previous trials.

All the contextual effects in the present experiments resulted from shifts in internal representations, and not from changes in the judgment functions. The present results, however, do not indicate whether the shifts in coded sensations are due to changes in the psychophysical stage or in the coding stage of stimulus processing (Figure 1). A purely sensory phenomenon that could be hypothesized to produce a context effect is sensory adaptation: A negatively skewed stimulus distribution contains many high concentrations and is more likely to produce adaptation than is a positively skewed distribution. Adaptation will lower the perceived intensities of the solutions.

In the present study, two measures were taken to avoid adaptation: a mouth rinse after each sample and an interstimulus interval ( 30 to $50 \mathrm{sec}$ ). Although these measures were perhaps not rigorous enough to restore the oral cavity to the preexperimental level (e.g., O'Mahony, 1972), the insignificant stimulus order effects in "homogeneous" comparisons suggests that they were effective in creating a constant background. In addition, adaptation cannot explain the present findings. First of all, adaptation affects the slope and form of psychophysical functions (e.g., Meiselman, 1968), whereas the psychophysical curves in the present study are essentially parallel. Furthermore, adaptation cannot account for the between-session transfer effect in Figure 7. In addition, Riskey (1982) has found similar context effects for three experimental conditions, differing in the degree of mouth rinsing and the duration of the interstimulus interval. It is concluded, therefore, that sensory adaptation cannot account for contextual effects.

\section{Is Water a Meaningful Zero Point on a Taste Intensity Scale?}

In Experiments 1, 3, and 4, different contexts caused roughly similar discrepancies in scale values for all the stimuli judged by naive subjects. Comparable parallelism was obtained by Mellers and Birnbaum (1982) for subjective darknesses of dot patterns (see their Figure 13). The parallelism suggests that changes in stimulus context cause shifts in the relative positions of several gustatory continua (sweetness, saltiness, water) before these are projected onto the target continuum specified in the task instructions.

If one presumes that water is a meaningful zero point on a taste intensity scale (De Graaf \& Frijters, 1988), then one is likely to conclude that stimulus context produces a shift in scale values of all the experimental stimuli. However, one can also choose another taste continuum and conclude that the scale value for water has shifted. The main argument for choosing the "water" dimension as the reference dimension is derived from chemistry and not from psychology: In a water stimulus, the tastant's concentration is zero. The adoption of the taste of water as zero point on a subjective taste intensity scale further implies that (1) water does not elicit the target taste intensity, and (2) the subjective impression elicited by tasting water is regarded as part of the target taste intensity continuum. Although water is not necessarily tasteless (e.g., McBurney \& Pfaffmann, 1963; McBurney \& Shick, 1971), experimenters can comply with the first demand by using experimental designs in which the probability of an undesired water taste is minimal. The second requirement, however, is not fulfilled in a universal way under all conditions. The present experiments have shown that the coupling between the scale value of water and the scale values of the target stimuli is context dependent. This finding, however, does not preclude the existence of valid ratio scales for taste intensity: The scale values calculated with water as the zero point could have ratio scale properties within a certain context.

\section{A Model for Context-Dependent Judgments}

The present and previous results (e.g., Marks \& Warner, 1991; Mellers \& Birnbaum, 1982; Rankin \& Marks, 1991) provide insight into the encoding process of the stimulus judgment sequence (Figure 1) for uni- and multidimensional stimulus sets. Four stages should be distinguished: 
Stimulus classification. First, perceived sensations are put into a limited number of classes. This classification occurs spontaneously, and the number and sizes of the classes used may depend on the subject or on task instructions. For the sense of taste, subjects can discriminate between several taste qualities (e.g., sweet, sour, bitter, salty, water). An important merit of the present study is that it has demonstrated that "water" or "no taste" forms a separate class.

Stimulus placement. For each stimulus, a place is determined on the continuum for that stimulus class. The size of each continuum is limited. The position on each continuum does not depend on stimulus frequency (positive-negative skew) or stimulus spacing. However, since the subjective continua are limited, stimulus placement is likely to depend on the size of the stimulus range. A larger range of perceived sensations narrows the interstimulus distances on the restricted continuum and thus decreases the slope of the psychophysical function.

Continuum placement. The relative positions of the subjective continua are determined. The process determining the relative continuum positions depends on stimulus frequency (Experiments 1-3) and relative stimulus range (Experiment 4). In addition, Rankin and Marks's (1991) data suggest that the size of contextual continuum shifts depends on the degree of qualitative similarity between the different continua. Similar continua are closely linked together and will be handled in similar ways when the relative positions of the continua are determined. Dissimilar continua are handled relatively independently and will, therefore, show large context effects (Schifferstein, 1994b).

Continuum projection. The task instruction (e.g., to judge total intensity or sweetness intensity) determines the way in which quality-specific continua are projected on the final continuum. The scale values on the final continuum then serve as input for cognitive integration processes and the judgment function (Figure 1).

An exhaustive investigation of contextual effects implies the adoption of a dynamic model of psychophysical judgment, in which previous experiences affect the formation of the current response. It challenges the feasibility of a classical, static approach using Fechner's (1860) or Stevens's (1955) law to estimate the psychophysical function for a type of stimulus, a sense quality, or a sense modality (e.g., Moskowitz, 1971; Stevens, 1955) while avoiding all kinds of "biases" (e.g., Poulton, 1989; Zwislocki \& Goodman, 1980). The variability and context dependency of momentary responses do not preclude the existence of a stable sensory basis for judgment. If systematic manipulation of experimental variables yields predictable shifts in responses, the underlying invariant structures - sensory scales - can be revealed (Birnbaum, 1974, 1978). Because the scale value for the blank stimulus shifts in relation to the scale values of the experimental stimuli with manipulations of experimental context, most continua lack a well-defined zero point (Birnbaum, 1978), which precludes the exis- tence of universal ratio scales and questions the validity of ratio scaling techniques (Birnbaum, 1978; Poulton, 1989). Consequently, the sensory scales for many continua have, at best, equal-interval properties. Possible exceptions are subjective length, area, and volume (Parker, Schneider, \& Kanow, 1975; Schneider \& Bissett, 1988). Irrespective of the sensory scale underlying psychophysical judgments, however, no theory of perceptual judgment is complete without an understanding of the functional dynamism due to the interplay between sensory and cognitive processes.

\section{REFERENCES}

ANDERSON, N. H. (1981). Foundations of information integration theory. New York: Academic Press.

Birnbaum, M. H. (1974). Using contextual effects to derive psychophysical scales. Perception \& Psychophysics, 15, 89-96.

BirnbaUM, M. H. (1978). Differences and ratios in psychological measurement. In N. J. Castellan, Jr., \& F. Restle (Eds.), Cognitive theory (Vol. 3, pp. 33-74). Hillsdale, NJ: Erlbaum.

Birnbaum, M. H., Parducci, A., \& Gifford, R. K. (1971). Contextual effects in information integration. Journal of Experimental Psychology, 88, 158-170.

De GraAf, C., \& FriJTers, J. E. R. (1988). Assessment of taste interaction between two qualitatively similar-tasting substances: A comparison between comparison rules. Journal of Experimental Psychology: Human Perception \& Performance, 14, 526-538.

De Graaf, C., Frijters, J. E. R., \& van Trijp, H. C. M. (1987). Taste interaction between glucose and fructose assessed by functional measurement. Perception \& Psychophysics, 41, 383-392.

De Graaf, C., Legger, A., \& Roozen, J. P. (1990). Anchored ratio scale values in the assessment of perceived sweetness, bitterness and chocolate flavour intensity in chocolate confetti and flakes. Journal of the Science of Food \& Agriculture, 50, 363-375.

FECHNER, G. T. (1860). Elemente der Psychophysik [Elements of psychophysics]. Leipzig: Breitkopf und Härtel.

FrIJTERS, J. E. R. (1993). Functional measurement in the study of mixture percepts. Chemical Senses, 18, 93-100

KLITZNER, M. D. (1975). Hedonic integration: Test of a linear model. Perception \& Psychophysics, 18, 49-54.

KroezE, J. H. A. (1983). Successive contrast cannot explain suppression release after repetitious exposure to one of the components of a taste mixture. Chemical Senses, 8, 211-223.

Marks, L. E. (1992). The contingency of perceptual processing: Context modifies equal-loudness relations. Psychological Science, 3 , 285-291

MARKS, L. E., \& WARNER, E. (1991). Slippery context effect and critical bands. Journal of Experimental Psychology: Human Perception \& Performance, 17, 986-996.

MCBridE, R. L. (1985). Stimulus range influences intensity and hedonic ratings of flavour. Appetite, 6, 125-131.

McBurney, D. H., \& Pfaffmann, C. (1963). Gustatory adaptation to saliva and sodium chloride. Journal of Experimental Psychology, 66, 523-529.

MCBurney, D. H., \& Shick, T. R. (1971). Taste and water taste of twenty-six compounds for man. Perception \& Psychophysics, 10 , 249-252.

Meiselman, H. L. (1968). Adaptation and cross-adaptation of the four gustatory qualities. Perception \& Psychophysics, 4, 368-372.

Mellers, B. A., \& Birnbaum, M. H. (1982). Loci of contextual effects in judgment. Journal of Experimental Psychology: Human Percep. tion \& Performance, 8, 582-601.

Moskowitz, H. R. (1971). Ratio scales of acid sourness. Perception \& Psychophysics, 9, 371-374.

O'MAhony, M. (1972). The interstimulus interval for taste: 1. The efficiency of expectoration and mouthrinsing in clearing the mouth of salt residuals. Perception, 1, 209-215. 
Parducci, A., Knobel, S., \& Thomas, C. (1976). Independent contexts for category ratings: A range-frequency analysis. Perception \& Psychophysics, 20, 360-366.

Parker, S., Schneider, B., \& Kanow, G. (1975). Ratio scale measurement of perceived lengths of lines. Journal of Experimental Psychology: Human Perception \& Performance, 1, 195-204.

Poulton, E. C. (1989). Bias in quantifying judgments. Hillsdale, NJ: Erlbaum.

Rankin, K. M., \& MARKs, L. E. (1991). Differential context effects in taste perception. Chemical Senses, 16, 617-629.

RISKEY, D. R. (1982). Effects of context and interstimulus procedures in judgments of saltiness and pleasantness. In J. T. Kuznicki, R. A. Johnson, \& A. F. Rutkiewic (Eds.), Selected sensory methods: Problems and applications to measuring hedonics (pp. 71-83). Philadelphia: American Society for Testing and Materials.

Riskey, D. R., Parducci, A., \& Beauchamp, G. K. (1979). Effects of context in judgments of sweetness and pleasantness. Perception $\&$ Psychophysics, 26, 171-176.

SCHIFFERSTEIN, H. N. J. (1994a). Contextual effects in the perception of quinine $\mathrm{HCl} / \mathrm{NaCl}$ mixtures. Chemical Senses, 19, 113-123.

SCHIFFERSTEIN, H. N. J. (1994b). Sweetness suppression in fructose/ citric acid mixtures: A study of contextual effects. Perception \& Psychophysics, 56, 227-237.

Schifferstein, H. N. J., \& Frijters, J. E. R. (1992a). Contextual and sequential effects on judgments of sweetness intensity. Perception \& Psychophysics, 52, 243-255.

SchiffersteIN, H. N. J., \& FriJters, J. E. R. (1992b). Sweetness does not habituate during a sip-and-spit experiment. Physiology \& $B e$ havior, 51, 331-337.
Schneider, B., \& Bissett, R. (1988). "Ratio" and "difference" judgments for length, area, and volume: Are there two classes of sensory continua? Journal of Experimental Psychology: Human Perception \& Performance, 14, 503-512.

SCHNEIDER, B., \& PARKer, S. (1990). Does stimulus context affect loudness or only loudness judgments? Perception \& Psychophysics, 48, 409-418

Stevens, S. S. (1955). The measurement of loudness. Journal of the Acoustical Society of America, 27, 815-829.

Stillman, J. A. (1993). Context effects in judging taste intensity: A comparison of variable line and category rating methods. Perception \& Psychophysics, 54, 477-484.

Vaughan, G. M., \& Corballis, M. C. (1969). Beyond tests of significance: Estimating strength of effects in selected ANOVA designs. Psychological Bulletin, 72, 204-213.

WARD, L. M. (1987). Remembrance of sounds past: Memory and psychophysical scaling. Journal of Experimental Psychology: Human Perception \& Performance, 13, 216-227.

WARD, L. M., \& LockHEAD, G. R. (1970). Sequential effects and memory in category judgments. Journal of Experimental Psychology, $\mathbf{8 4}$ 27-34.

ZWislockl, J. J., \& GoOdman, D. A. (1980). Absolute scaling of sensory magnitudes: A validation. Perception \& Psychophysics, 28, 28-38.

(Manuscript received February 8, 1994; revision accepted for publication August 22, 1994.) 